\title{
NEPHROTOXICITE ASSOCIEE AU METHOXYFLURANE
}

\author{
ANDré Lapointe, M.D., F.R.C.P.(c), et Natalis Bele-Binda, M.D. *
}

DEPUIS QUELQUes aNNÉES, l'attention des anesthésiologistes a été attirée par la publication de quelques articles relatifs à la néphrotoxicité du méthoxyflurane (Penthrane).$^{1-8}$ Cette néphrotoxicité apparait dans les 24 heures qui suivent une anesthésie au méthoxyflurane. On l'appelle communément la polyurie au Penthrane. Certains auteurs l'ont qualifiée de diabète insipide néphrogénique ou de diurèse hyposthénurique postopératoire.

Cliniquement, la néphrotoxicité associée au méthoxyflurane se manifeste par des signes d'insuffisance rénale à haut débit. Ce qui la caractérise surtout, c'est que dans les premiers jours qui suivent une anesthésie au méthoxyflurane, le malade présente une diurèse marquée qui peut varier entre $2,600 \mathrm{ml}$ et 12,850 $\mathrm{ml}$ par 24 heures. Rapidement s'installe un tableau de déshydratation sévère avec perte de poids importante due au déficit de la balance liquidienne. Parfois, on note de la confusion mentale. Fait caractéristique, cette polyurie attribuée au méthoxyflurane ne répond pas à linjection d'A.D.H. exogène.

L'urine est de faible densité entre 1002 et 1005 . L'osmolalité urinaire est abaissée et tend à rejoindre celle du plasma; dans certains cas, elle est même inférieure à celle du plasma.

Dans le sang, l'osmolarité plasmatique est élevée. Il y augmentation de la natrémie, de l'azotémie, de l'acide urique, de l'azote uréique et de la créatinine. Le potassium et le calcium sanguins restent dans les limites de la normale. Cependant, nous avons noté, de façon isolée, une élévation de calcium sérique chez un de nos patients (Tableau V). Sur le plan évolutif, ce tableau a une durée moyenne de 15 jours et se termine généralement par une récupération spontanée.

\section{Historique}

La néphropathie associée au méthoxyflurane n'est pas un syndrôme nouveau. Il semble que déjà lors des études expérimentales qui ont précédé la mise sur le marché du méthoxyflurane on ait observé certains cas d'insuffisance rénale. En 1964, Paddock ${ }^{1}$ rapporte trois cas d'insuffisance rénale à la suite d'anesthésie au méthoxyflurane; deux de ces patients sont morts en hyperazotémie mais le troisième a survécu avec une fonction rénale déficiente. La même année, Artusio confie à Paddock que trois de ses patients avaient présenté de la polyurie après une anesthésie au méthoxyflurane.

En 1966, Crandell, un chirurgien, ${ }^{2-3}$ surprit les anesthésiologistes en revelant que chez 94 patients anesthésiés au méthoxyflurane, 16 (soit 17 pour cent) ont

-Dr Lapointe est Anesthésiologiste, Assistant dans le Service d'anesthésie et de réanimation de l'Hôtel-Dieu de Québec. Dr. Bele-Binda est Anesthésiologiste, Résident étranger en anesthésie et réanimation à l'Hôtel-Dieu de Québec, Boursier de l'O.M.S.

Canad. Anaesth. Soc. J., vol. 17, no. 2, March 1970 
développé une néphropathie attribuée à cet agent anesthésique. Cette incidence élevée de néphropathie reliée au méthoxyflurane trouve un appui dans l'article de Pezzi et ses collaborateurs ${ }^{4}$ qui rapportent 20 cas ( 16 pour cent) de polyurie associée au méthoxyflurane sur 123 cas d'anesthésie au méthoxyflurane en chirurgie abdominale dont six cas mortels.

Depuis le travail de Crandell, quelques auteurs ont rapporté d'autres cas de néphropathie associée au méthoxyflurane. Austin ${ }^{5}$ raconte avoir observé six cas de polyurie dont cinq reliés à l'administration du méthoxyflurane et 1 à l'éther. Snyder ${ }^{6}$ en a rapporté trois cas. Elkington ${ }^{7}$ décrit un cas de polyurie au méthoxyflurane avec atteinte hépatique chez un patient obèse; cette double atteinte rénale et hépatique était attribuée à l'administration du méthoxyflurane. Lebowitz ${ }^{8}$ publie deux cas d'atteinte rénale reliée au méthoxyflurane dont un des patients était porteur d'un diabète léger.

Devant l'incidence fort élevée de néphropathie associée au méthoxyflurane notée par Crandell et corroborée par Pezzi, nous avons fait un relevé des cas d'anesthésie au méthoxyflurane pratiquée à l'Hôtel-Dieu de Québec.

De 1960 au $1^{\text {er }}$ mai 1969, nous avons pratiqué 20,825 anesthésies au méthoxyflurane comportant 17,335 anesthésies pour chirurgie générale et 3,490 anesthésies obstétricales comme le montre le Tableau I. Au cours de cette période de neuf ans, nous avons observé cinq cas de néphropathie attribuée à l'anesthésie au méthoxyflurane. Si nous excluons du calcul les 3,490 anesthésies obstétricales dont la durée moyenne n'excède pas 30 minutes, la fréquence est donc de cinq sur 17,335 , soit 0.02884 pour cent.

TABLEAU I

\begin{tabular}{lcc}
\hline \hline Année & Chirurgie générale & Obstétrique \\
\hline 1960 & 21 & 0 \\
1961 & 2,833 & 365 \\
1962 & 3,781 & 488 \\
1963 & 3,085 & 467 \\
1964 & 2,231 & 461 \\
1965 & 2,255 & 401 \\
1966 & 2,796 & 471 \\
1967 & 1,666 & 348 \\
1968 & 1,611 & 361 \\
1969 (jusqu'au & 546 & 128 \\
\multicolumn{2}{c}{ 1or mai 1969) } & \\
Total en chirurgie générale & 17,335 \\
Total en obstétrique & 3,490 \\
Total général & 20,825 \\
\hline
\end{tabular}

RAPPont DES CAS

Cas no. 1 (voir Tableau II)

Il s'agit de Madame F.J., 71 ans, dossier numéro 73218. Cette patiente obèse pesait 174 livres $(79 \mathrm{~kg}$ ) à l'entrée. Son poids habituel est de 180 livres (81.80 $\mathrm{kg}$ ). Les antécédents sont sans particularité. Les examens à l'entrée, le 10 avril 1967, se lisent comme suit: Hb 13.6 gm pour cent; groupe sanguin $3 \mathrm{~B}$, Rh positif; T.A. 180/90; pouls 70. Urée sanguine 0.33 ; azote uréique 0.155 . Densité urinaire 


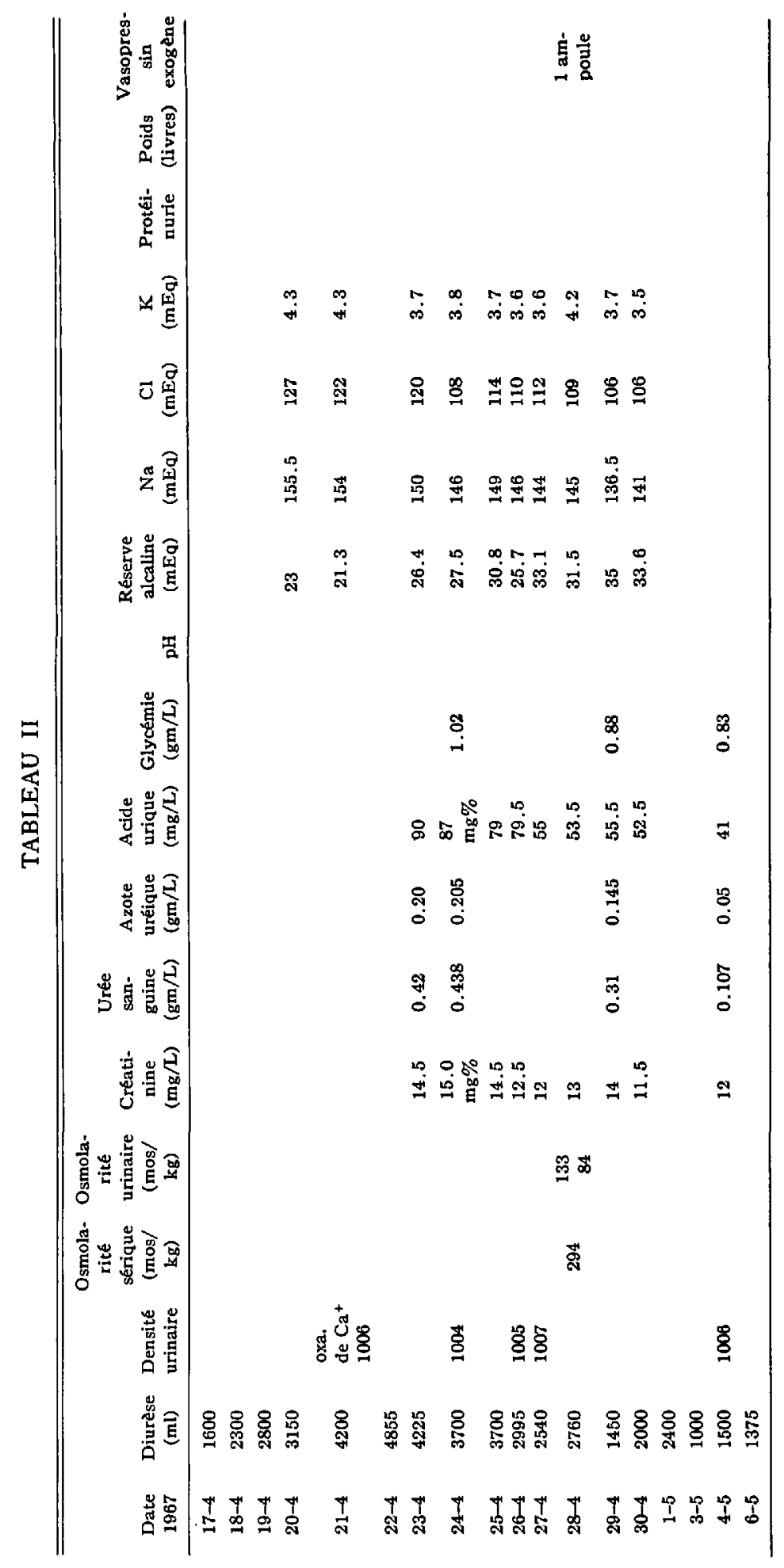


1024; BSP après 45 minutes 3.6 pour cent ( $\mathrm{N}$ moins de 6 pour cent). ECG - bloc de branche droit avec déviation axiale gauche.

La patiente fut hospitalisée pour des troubles intestinaux qui ont amené la découverte d'un épithélioma du sigmoïde. Le 17 avril 1967, elle subit une résection du sigmoïde après un cathétérisme urétéral bilatéral sous anesthésie générale.

L'anesthésie a été maintenue avec méthoxyflurane, $\mathrm{N}_{2} \mathrm{O}, \mathrm{O}_{2}$, après une induction au Valium $20 \mathrm{mg}$ i.v. suivie de l'intubation sous Syncurine (décaméthonium) $2 \mathrm{mg}$, et Anectine (succinylcholine) $40 \mathrm{mg}$. Le relâchement musculaire a été obtenu avec de la gallamine (Flaxedil) $120 \mathrm{mg}$ au cours des cinq heures que dura l'anesthésie. La prémédication a été assurée avec $75 \mathrm{mg}$ de Démérol (péthidine) et $0.40 \mathrm{mg}$ d'atropine 45 minutes avant l'intervention. L'anesthésie s'est déroulée normalement sans chute tensionnelle importante. Une transfusion de $450 \mathrm{ml}$ a été donnée sans incident pendant l'intervention.

Avant l'intervention, la malade reçut Declomycine $150 \mathrm{mg}$ per os par jour, Sulfatholidine $1.50 \mathrm{mg}$ per os par jour et Librium, $50 \mathrm{mg}$ i.m.

Dès le soir de l'intervention, la malade développa une polyurie progressive (1600 $\mathrm{ml}$ le soir même) dépassant largement l'apport joumalier des liquides avec apparition d'une soif intense, d'une hypernatrémie avec déshydratation. L'urine est hypotonique, la densité urinaire restant basse: 1006. La malade est restée lucide, de bonne humeur se plaignant seulement de soif. Ce tableau suggère une atteinte rénale empêchant la réabsorption d'eau au niveau des tubules consécutives à l'administration du méthoxyflurane à moins qu'il ne s'agisse d'une atteinte de l'hypophyse postérieure par métastase néoplasique. Un test de privation d'eau associé au test de suppression à l'A.D.H. pratiqué le 25 avril 1967 donne un résultat discordant; en effet, alors que le débit urinaire a semblé diminué, la densité urinaire est demeurée basse (1002) ce qui fait conclure qu'il n'y a pas eu de réponse positive à l'injection de Pitressin d'où forte suspicion d'atteinte rénale.

En l'absence de vomissements, de constipation, de troubles neuromusculaires, d'anoxie, le diagnostic d'hypercalcémie ne peut facilement être envisagé.

En post-opératoire immédiat, la malade reçut Librium 50, Démérol 75, Gravol, Penbritine $1 \mathrm{gm}$ dans le sérum.

\section{Cas no. 2 (voir Tableau III)}

L'Abbé M.P., 65 ans, dossier numéro 5605. Admis le 29 janvier 1968 à l'HôtelDieu de Québec pour cholélithiase chronique. Dans les antécédents: I Familiaux: 1 frère diabétique, 1 oncle diabétique. Ir Personnels: diabète léger traité par Diabinèse, $1 / 2$ comprimé 2 fois par jour. Poids à l'entrée, 152 livres (69 kg).

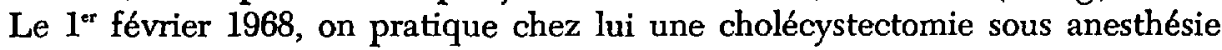
générale au méthoxyflurane d'une durée d'une heure 45 minutes. Détails de l'anesthésie: prémédication, Atropine $0.40 \mathrm{mg}$, Benadryl $25 \mathrm{mg}$. Induction, Pentothal $500 \mathrm{mg}$, Syncurine $2 \mathrm{mg}$, Anectine (succinylcholine) $40 \mathrm{mg}$. Maintien, $\mathrm{N}_{2} \mathrm{O}, \mathrm{O}_{2}$, méthoxyflurane-Flaxedil $100 \mathrm{mg}$. Décurarisation, Atropine $0.40 \mathrm{mg}$, Prostigmine $1 \mathrm{mg}$. On installe chez lui en peropératoire, un soluté glucosé 5 pour cent dans lequel on laisse couler lentement de l'insuline Toronto 12 unités. Les suites opératoires immédiates se déroulent normalement à part une diurèse 


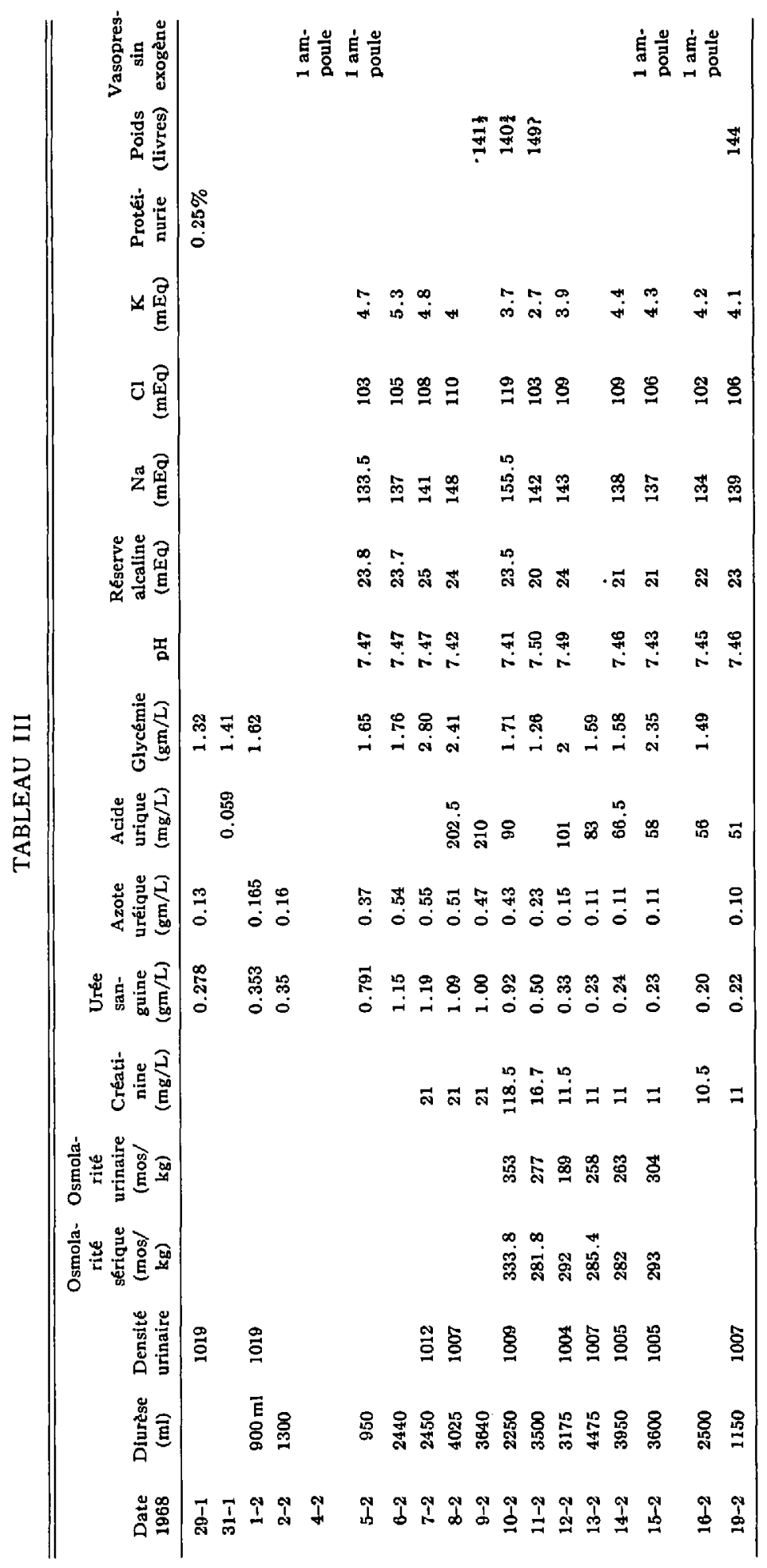




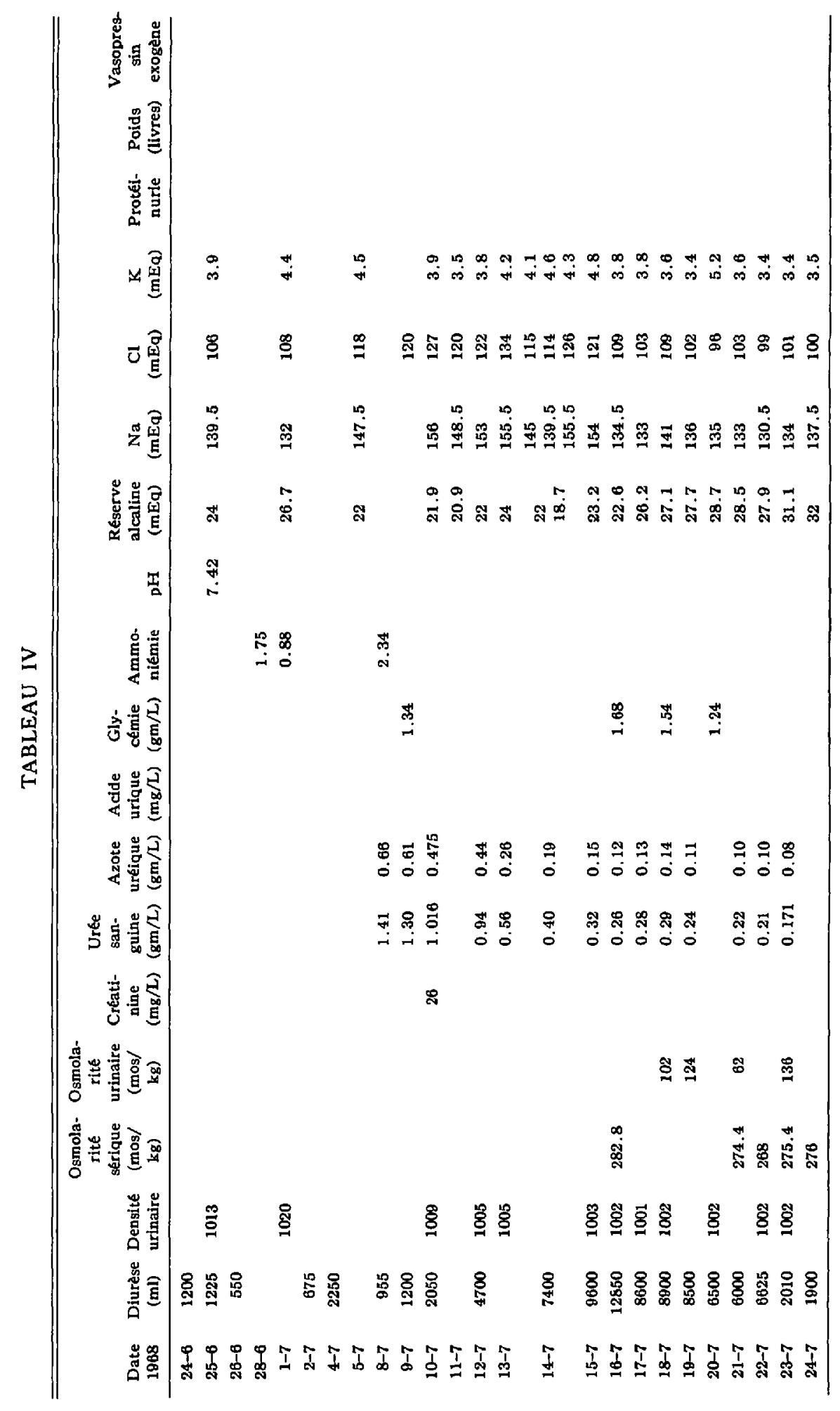


qui s'annonce abandante: $900 \mathrm{ml}$ le soir de l'intervention et $1300 \mathrm{ml}$ le lendemain matin.

Le 4 février 1968, on décide de lui faire un test à l'A.D.H. (Pitressin). On répète le test le 5 février 1968, jour où le malade est réopéré pour une obstruction intestinale. De nouveau l'anesthésie est maintenue au méthoxyflurane pour une durée de trois heures. On pratique cette fois-ci une anastomose iléo-coecale.

Dans les suites opératoires, on note un bilan liquidien négatif avec déshydratation (langue sèche et rotie, pli cutané important et généralisé), perte pondérale (152 livres le 29 janvier 1968, 141\% livres le 9 février 1968), des modifications électrolytiques progressives, le $\mathrm{Cl}$ passe de 103 à $119 \mathrm{mEq} / \mathrm{L}$ en quatre jours et le $\mathrm{Na}$ de $133.5 \mathrm{mEq} / \mathrm{L}$ à $155.5 \mathrm{mEq} / \mathrm{L}$. Le patient devient confus, obnubilé. Entretemps, le malade présente une diarrhée, ce qui aggrave les troubles électrolytiques.

\section{Cas no. 3 (voir Tableau IV)}

Madame L.G., 41 ans, dossier numéro 100762. Cette patiente porteuse d'un ulcus duodénal fut admise le 24 juin 1968 à l'Hôtel-Dieu de Québec pour hémorragie digestive (hématémèse) suivie d'un état de choc. Son poids à l'entrée est de 143 livres ( $65 \mathrm{~kg}$ ).

Le 2 juillet 1968, elle subit une vagotomie avec gastrectomie subtotale (Billroth $\mathrm{I}$ ), réparation de hernie diaphragmatique et appendicectomie de principe. Détails de l'anesthésie: induction, Pentothal $500 \mathrm{mg}$; intubation sous Syncurine 2 $\mathrm{mg}$, Anectine (succinylcholine) $40 \mathrm{mg}$; maintien de l'anesthésie, méthoxyflurane$\mathrm{N}_{2} \mathrm{O}, \mathrm{O}_{2}$; durée de l'anesthésie, trois heures. L'anesthésie s'est déroulée sans heurts; en postopératoire, la malade a reçu Mécholine $0.5 \mathrm{ml}$ sous cutanée.

Le 3 juillet, on répète Mécholine $0.5 \mathrm{ml}$ sous cutanée deux fois dans les 24

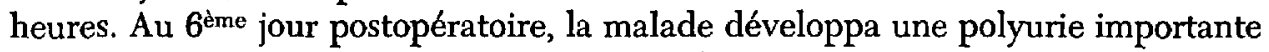
avec déshydratation et ralentissement mental. Le temps de Quick est de 41 pour cent avec un taux de céphaline à 22 pour cent. Les 9 et 10 juillet 1968, soit 8 jours après l'intervention, on met en évidence dans les urines des cristaux d'oxalate de calcium.

\section{Cas no. 4 (voir Tableau V)}

Monsieur G.P., 38 ans, dossier numéro 89886. Comptable de 38 ans, obèse: 190 livres $(86 \mathrm{~kg} \mathrm{366}$ ) a déjà pesé 225 livres (102 kg). Reconnu diabétique, il est traité au Diabinèse depuis l'âge de 21 ans. Admis le 31 octobre 1968 à l'HôtelDieu de Québec pour ulcus duodénal, il est immédiatement traité avec insuline N.P.H.

Le 6 novembre 1968, il subit une gastrectomie (Billroth II). L'anesthésie comporte $500 \mathrm{mg}$ de Pentothal pour l'induction, Syncurine $2 \mathrm{mg}$, Anectine (succinylcholine) $40 \mathrm{mg}$ pour l'intubation. Le maintien de l'anesthésie, qui dure deux heures et 40 minutes, se fait avec méthoxyflurane et gallamine. On réintervient immédiatement à cause d'un décompte de gaze inexact. Cette fois, linduction est faite au Pentothal $400 \mathrm{mg}$ et au Valium (diazépam) $10 \mathrm{mg}$; le maintien au méthoxyflurane- $\mathrm{N}_{2} \mathrm{O}, \mathrm{O}_{2}$. La durée de la deuxième anesthésie est de 30 minutes. 


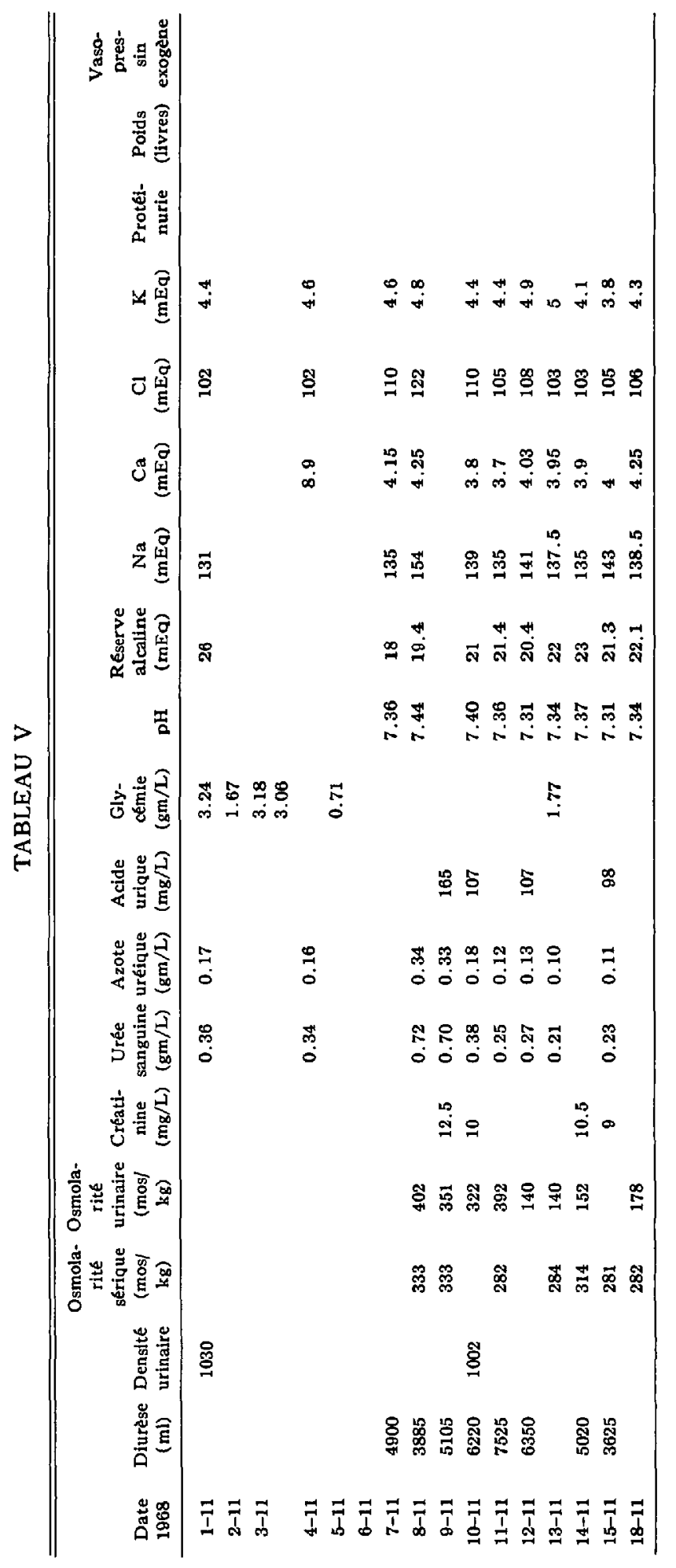




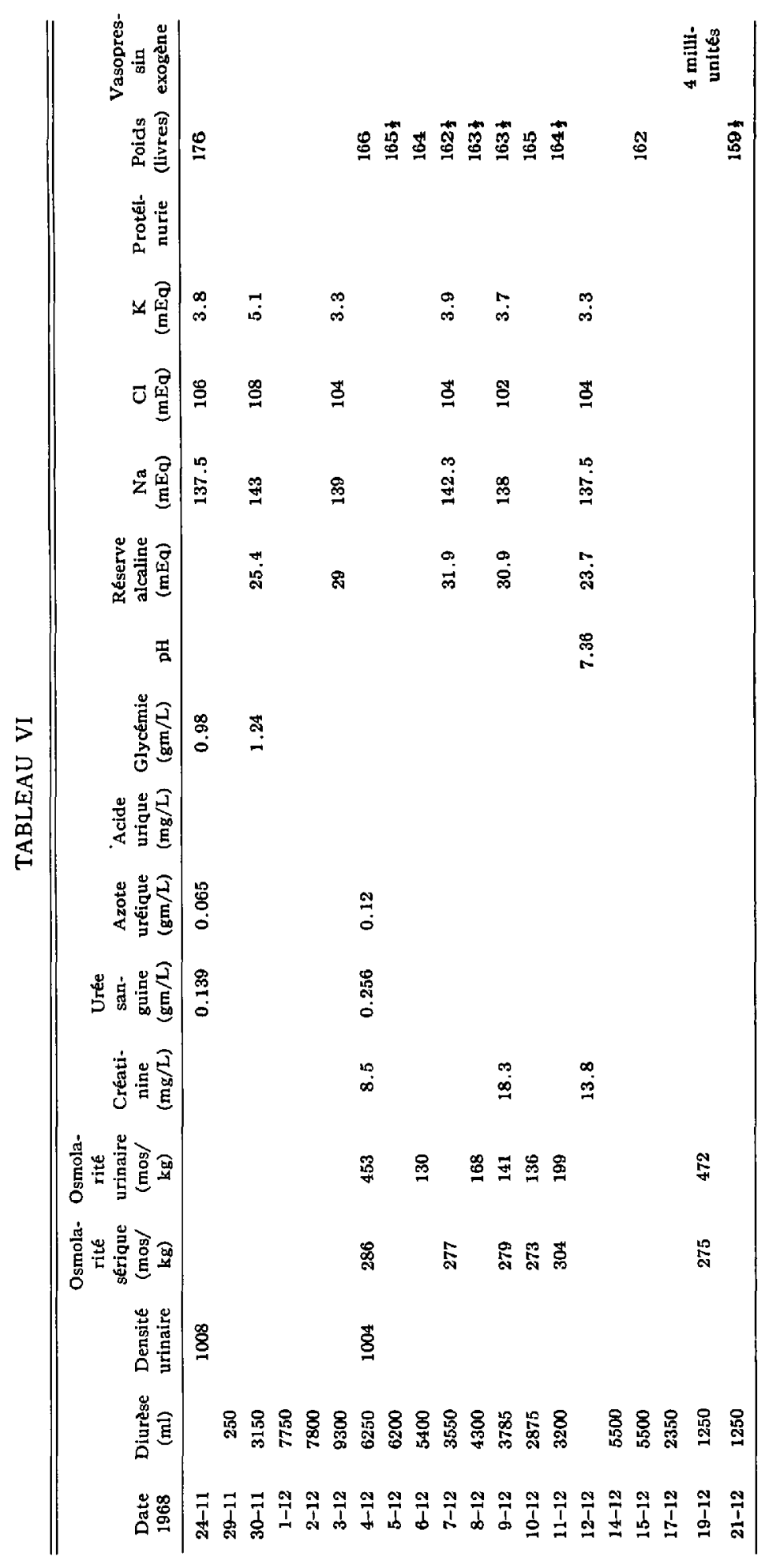


Dans la salle de réveil, la glycémie est de $3.42 \mathrm{gm}$. Le malade présente une polyurie dès les premiers jours postopératoires.

\section{Cas no. 5 (voir Tableau VI)}

Madame L.G., 48 ans, dossier numéro 110309. Patiente obèse: 176 livres à l'entrée $(80 \mathrm{~kg}$ ) traitée depuis deux ans avec des hypoglycémiants oraux pour diabète sucré. Admise le 24 novembre 1968 à l'Hôtel Dieu du Québec pour cure d'éventration consécutive à une réparation dhernie ombilicale.

Examen à l'entrée: polyurie, polydipsie, gros appétit, baisse de l'acuité visuelle. Objectivement: hirsutisme, varices des membres inférieurs, potomanie, éventration abdominale. $\mathrm{Hb} 13.45 \mathrm{gm}$ pour cent, groupe sanguin $2 \mathrm{~A} \mathrm{Rh}$ positif, T.A. $155 / 90$, pouls 72 à la minute. Urée sanguine 0.139 , azote uréique 0.065 , glycémie 0.98 , hyperglycémie provoquée normale. Densité urinaire 1008. ECG anomalie de la phase terminale non spécifique. La radiographie de la selle turcique est normale.

Le 29 novembre 1968, on procède à la réparation de l'éventration abdominale sous anesthésie générale au méthoxyflurane. Durée de l'anesthésie, 3 heures. Après prémédication au Démérol (pethidine) $75 \mathrm{mg}$, Atropine $0.40 \mathrm{mg}$, l'induction est pratiquée avec $275 \mathrm{mg}$ de Pentothal et l'intubation après Syncurine $2 \mathrm{mg}$, Anectine $40 \mathrm{mg}$. Le maintien est assuré avec de $\mathrm{N}_{2} \mathrm{O}, \mathrm{O}_{2}$, méthoxyflurane, Flaxedil $120 \mathrm{mg}$. L'ECG peranesthésique est normal; une transfusion isogroupe de $450 \mathrm{ml}$ est bien supportée.

La décurarisation est faite avec $0.40 \mathrm{mg}$ d'Atropine et $1 \mathrm{mg}$ de Prostigmine. Un dosage des 11 hydroxycorticostéroïdes libres (cortisol) effectué le 3 décembre 1968 donne le résultat suivant: à 8 heures $270 \mathrm{mg} / \mathrm{L}$ (123 à 310), à 15 heures $209 \mathrm{mg} / \mathrm{L}$ soit un abaissement de 22.6 pour cent - l'abaissement normal est de 22 à 54 pour cent.

Dans les suites opératoires immédiates, la malade a reçu $0.4 \mathrm{mg}$ de Cédilanid deux fois à la salle de réveil, de l'Achromycine en perfusion veineuse, du Darvon et du Lévo-dromoran les jours suivants.

\section{CommentaIRES}

Deux faits ont attiré notre attention dans l'apparition du syndrôme polyurique après administration de méthoxyflurane:

$1^{\circ}$ La prééxistance d'un diabète léger se rencontre chez trois de nos patients et la présence d'une obésité est notée chez un autre. Elkington et ses collaborateurs ont d'ailleurs souligné le rôle probable de l'obésité dans la pathogénie de la néphropathie après penthranisation. Depuis cette constatation, nous adoptons une attitude abstentionniste tant que faire se peut en présence d'un diabète si léger soit-il ou d'une obésité. Un fort pourcentage de nos anesthésies pratiquées en O.R.L. l'ont été chez des enfants et nous avons été frappés par l'absence d'atteinte rénale chez ces petits patients; ce qui semble confirmer la notion du terrain de prédisposition.

$2^{\circ}$ La durée de l'anesthésie semble un facteur dominant. En effet, sur les 3,490 anesthésies obstétricales qui sont des anesthésies de courte durée, nous n'avons 
noté aucun cas de néphropathie et à notre connaissance, aucun cas de ce genre n'a été rapporté. Le facteur durée d'anesthésie, nous a même incités par souci d'objectivité à n'établir le pourcentage des cas de néphropathie dans notre travail qu'en nous basant sur les anesthésies de plus d'une heure excluant de ce fait les anesthésies obstétricales. Ainsi, la fréquence de 0.02884 pour cent rapportée plus haut, a été calculée sur 17,335 anesthésies seulement et non sur les 20,825 de notre série totale.

Ce faible pourcentage de 0.02884 pour cent nous place loin de celui de 17 pour cent noté par Crandell et corroboré par Pezzi. Loin de nous l'idée de mettre en doute les craintes de Crandell. Cette basse incidence nous permet quand même de ne pas trop redouter l'éventualité d'une néphropathie au méthoxyflurane tout en ayant présent à l'esprit que les anesthésies prolongées surtout lorsqu'il existe la notion d'obésité et de diabète semblent prédisposer le patient à cette éventualité.

\section{RÉSUMÉ}

Le travail que nous présentons, après une brève revue de la littérature, a pour but de faire ressortir la faible incidence de néphrotoxicité associée au méthoxyflurane (Penthrane) notée à l'Hôtel-Dieu de Québec.

En effet, sur 17,335 anesthésies au méthoxyflurane de plus d'une heure, nous n'avons observé que 5 cas de néphrotoxicité associée au méthoxyflurane, ce qui en pourcentage représente une incidence de $<0.03$ pour cent.

Ce travail nous a permis de mettre en relief l'importance du facteur durée d'anesthésie, la prédisposition possible constituée par le diabète et l'obésité et Pabsence du syndrôme en anesthésie obstétricale et infantile.

\section{SUMmary}

The present study, after a brief review of the literature, points out the small incidence of nephrotoxicity associated with methoxyflurane (Penthrane) seen at l'Hôtel-Dieu de Québec. Of 17,335 anaesthetics with methoxyflurane lasting more than one hour we have observed only five cases of renal toxicity, representing an incidence of less than 0.03 per cent.

This study has permitted us to place in focus the importance of the factor of duration of anaesthesia, the possible predisposition in the diabetic and the obese, and the absence of the syndrome in obstetrical anaesthesia and infants.

\section{BIBLIOGRAPHIE}

1. Paddock, R. B.; Pafker, J. W.; \& Guadagni, N. P. The Effects of Methoxyflurane on Renal Function. Anesthesiology. 25: 707 ( 1964).

2. Crandell, W. B.; Pappas, S. G.; \& Macdonald, A. Nephrotoxicity Associated with Methoxyflurane Anesthesia. Anesthesiology. 27: 591 (1966).

3. Crandell, W. B. \& Macdonaid, A. Nephropathy Associated with Anesthesia: A Follow-up Report. J.A.M.A. 205: 798 (1968).

4. Pezzi, P. J.; Frobese, A. S.; \& Greenberg, S. R. Methoxyflurane and Renal Toxicity. Lancet. 1: 823 (1966). 
5. Austin, W. H. \& VI.LANDry, P. J. Methoxyflurane and Renal Function. (Correspondence.) Anesthesiology. 28: 637 (I967).

6. SNyder, R. D.; McCall, D. M.; \& Hayes, M. A. Functional Defect in Post-operative Polyuric Renal Failure. Surg. Forum. 17: 19 (1966)

7. Elkington, S. G.; Goffinet, J. A.; \& Conn, H. O. Renal and Hepatic Injury Associated with Methoxyflurane Anesthesia. Ann. Intern. Med. 69: 1229 (1968)

8. Lebowitz, M. H. Nephrogenic Diabetes Insipidus Following Methoxyflurane Anesthesia: A Report of Two Cases, Anesth. \& Analg. 2: 233 (1969). 